\title{
Effects on the Maternofetal Unit of the Rabbit Model after Substitution of the Amniotic Fluid with Perfluorocarbons
}

\author{
Oliver J. Muensterer ${ }^{\mathrm{a}}$ Verena J. Klis $^{\mathrm{a}}$ Holger Tilla ${ }^{\mathrm{a}}$ Dietrich von Schweinitz ${ }^{\mathrm{a}}$ \\ Georg Simbruner ${ }^{b}$
}

Departments of a Pediatric Surgery, Dr. von Hauner Children's Hospital, Munich, Germany, and ${ }^{b}$ Neonatology, Leopold Franzens University, Innsbruck, Austria

\section{Key Words}

Perfluorocarbon · Fetal endoscopic surgery · Amniotic fluid $\cdot$ Rabbit model

\begin{abstract}
Objectives: Exchanging amniotic fluid (AF) with perfluorocarbon (PFC) may serve as a medium for fetoscopic surgery. This study evaluates the distribution and physiologic effects of intraamniotic PFC as a medium for fetoscopy. Methods: Fetuses of 17 pregnant rabbits underwent either exchange of the AF with PFC, electrolyte solution (ES), or control. The quality of vision during fetoscopy was assessed in AF and PFC. After 6 h, we determined the distribution of PFC in the maternofetal unit. Results: Quality of vision during fetoscopy was better in PFC than with AF. There was no difference in fetal survival between the study groups. PFC was demonstrated on X-ray in the pharynx of 4 fetuses, and the esophagus in 1. Conclusions: PFC provided an ideal medium for fetoscopy without fetal compromise.
\end{abstract}

Copyright (C) 2005 S. Karger AG, Basel

\section{Introduction}

Over the past decade a large number of diagnostic and therapeutic interventions of the fetus have been proposed and introduced $[1,2]$. Fetal intraamniotic endoscopy has been used to assess and surgically correct malformations with varying success $[3,4]$. During fetoscopy, intraamniotic hemorrhage or meconium may reduce or abolish vision. Continuous replacement of clouded amniotic fluid with a clear one is not feasible. Perfluorocarbons are biologically inert, fully transparent fluids with a specific weight approximately double that of water. They form a distinct interface with aqueous solutions. Consequently, any fluid or debris would float to the top and keep the perfluorocarbon (PFC) medium clear for fetoscopic interventions. However, higher intrauterine pressure and distension of the uterus may elicit uterine contractions [5]. Instillation of PFC or exchanging amniotic fluid with PFC might also compromise the fetus and decrease its viability.

PFC filled into ventilated lungs of animals has been shown to be absorbed and distributed to various organs, in particular in tissue containing phospholipids such as the brain [6]. The long-term effects of PFC on cell function and development is not known. In fact, almost nothing is known on the various effects of PFC instilled into the intrauterine cavity, on doe, uterus and fetus.

We therefore investigated whether exchanging amniotic fluid with PFC would result (a) in fetal compromise or death, (b) whether an amnion cavity filled with PFC would allow better vision during fetoscopy than amniotic fluid, and (c) how the intraamniotic PFC would be distributed within the doe and fetus.

\section{KARGER}

Fax +41613061234 E-Mail karger@karger.ch www.karger.com
(C) 2005 S. Karger AG, Basel

$1015-3837 / 05 / 0205-0359 \$ 22.00 / 0$

Accessible online at: www.karger.com/fdt
Oliver J. Muensterer, MD

Department of Pediatric Surgery, Dr. von Hauner Children's Hospital University of Munich, Lindwurmstrasse 4, DE-80337 Munich (Germany) Tel. +49 8951602811 , Fax +498951604726

E-Mail oliver.muensterer@med.uni-muenchen.de 


\section{Methods}

The study protocol was approved by the Bavarian government commission for animal research.

\section{Anesthetic Technique}

After premedication with $10 \mathrm{mg}$ of s.c. diazepam (Diazepamratiopharm ${ }^{\circledR}$, ratiopharm $\mathrm{GmbH}$, Ulm, Germany), anesthesia was induced in 17 time-mated, pregnant New Zealand White rabbits (Harlan Winkelmann, Borchen, Germany) using 40 mg s.c. midazolam (Dormicum, Hoffmann-La Roche AG, Grenzach, Germany), 0.4 mg i.m. medetomidin (Dormitor, Pfizer GmbH, Karlsruhe, Germany), 40 mg i.m. ketamine (Ketanest, Parke-Davis $\mathrm{GmbH}$, Freiburg, Germany), and $0.5 \mathrm{mg}$ s.c. atropine sulfate (SigmaAldrich GmbH, Deisenhofen, Germany). As soon as the corneal reflexes were absent, intubation was performed with a straight blade laryngoscope and a 3.5 uncuffed endotracheal tube.

The abdomen, chest, ears and left upper arm were shaved. The doe was monitored by electrocardiogram, pulse oximetry from the left upper arm, and a rectal temperature probe. Body temperature was maintained between 37.0 and $38.0^{\circ} \mathrm{C}$ throughout the experiment by placing the animal on an adjustable warming blanket. Two 22-gauge catheters were introduced into the ear vein and artery for intravenous access and monitoring of blood gases. Dextrose solution $5 \%$ with $\mathrm{NaCl} 52 \mathrm{~m} M / 1$ was administered at $4 \mathrm{ml} / \mathrm{kg} / \mathrm{h}$. Anesthesia was maintained during the experiment by continuous i.v. infusion of $80 \mu \mathrm{g} / \mathrm{kg} / \mathrm{min}$ of ketamine and $4 \mu \mathrm{g} / \mathrm{kg} / \mathrm{min}$ of midazolam, titrated to adequate sedation level as needed. Opiates were deliberately not used to avoid fetal respiratory depression and thereby impede aspiration of intraamniotic fluids, as administration of opiates have been shown to cause apnea in fetal sheep [7]. Tocolysis was achieved by continuous i.v. administration of terbutaline (Bricanyl, AstraZeneca, Wedel, Germany) $2 \mu \mathrm{g} / \mathrm{kg} / \mathrm{min}$ with $2 \mathrm{mg}$ s.c. boluses before each manipulation of the uterus including ultrasound checks of fetal heart rate. The doe was ventilated by pressure-controlled intermittent mandatory ventilation to maintain an arterial $\mathrm{pCO}_{2}$ between 40 and $50 \mathrm{~mm} \mathrm{Hg}$. Maternal arterial blood gases were checked at hourly intervals.

\section{Subjects and Study Design}

The intervention was performed on gestational day 27 (term 31 days). After identifying the number of viable fetuses by fetal echocardiography, 3 fetuses were randomly selected and assigned to one of three groups: (1) amniotic fluid exchanged with PFC (PFC group), (2) amniotic fluid exchange with electrolyte solution (ES group), or (3) no manipulation at all to serve as a control group. To evaluate the applicability of different PFC compounds, four similar types of PFC liquid were used (Pharmpur GmbH, Augsburg, Germany): perfluorodecalin, perfluorooctane, perfluoro (1,3-dimethylcyclohexane) in 2 fetuses each, and perfluorooctylbromide (PFOB) in 11 fetuses (the main difference of these agents is the comparably high radiopacity of PFOB compared to the others). To assess viability of the fetuses, fetal heart rate was monitored by echocardiography at hourly intervals using a 5-mHz linear ultrasound probe (Sonoline AC, Siemens AG, Erlangen, Germany), visualizing the fetal heart and counting heart beats over $1 \mathrm{~min}$. Survival was defined as having a heart rate $\geq 100 / \mathrm{min}$. While the uterus had to be exposed for echocardiographic measurements, it remained inside the maternal abdomen, closing the midline laparotomy intermittently with four sharp towel clamps. Besides the designated control fetus, the other unmanipulated littermates were followed macroscopically, but did not undergo regular echographic evaluation to limit the intervals during which the uterus was exposed. Intraamniotic pressure was not directly measured.

\section{Surgical Technique}

To exchange the amniotic fluid with PFC, a 6-0 polypropylene pursestring suture was placed through the myometrium, chorionic and amniotic membranes at the region just anterior to the fetal neck, which was easily identified through gentle palpation. A $22-$ gauge intravenous catheter with four small precut perforations along the length of the sheath was advanced into the amniotic cavity through the pursestring suture. To stain the amniotic fluid for easier identification during the exchange process, $0.5 \mathrm{ml}$ of a $0.1 \%$ methylene blue solution was instilled through the catheter. The blue-stained lighter amniotic fluid was then replaced gradually with $5 \mathrm{ml}$ of denser, clear PFC using aliquots of $0.5 \mathrm{ml}$ at a time, thereby filling the amniotic cavity from the bottom up. After complete exchange, the catheter was withdrawn, pulling the pursestring suture tight to accomplish a watertight closure of the membranes and the uterine wall.

Subsequently, the amniotic fluid of the sibling assigned to the ES group was exchanged with $5 \mathrm{ml} \mathrm{ES}\left(\mathrm{Na}^{+} 140 ; \mathrm{K}^{+} 5 ; \mathrm{Ca}^{2+} 2.5\right.$; $\left.\mathrm{Mg}^{2+} 1.5 ; \mathrm{Cl}^{-} 153 \mathrm{mmol} / \mathrm{l}\right)$ in exactly the same manner. The third fetus selected for control remained untouched.

At the end of the experiment, $6 \mathrm{~h}$ after amniotic fluid exchange, the does were killed by an i.v. bolus of $20 \mathrm{~m} M$ potassium chloride. The vagina and adnexae were ligated and the uterus excised in toto.

In order to evaluate visibility and intraoperative handling during fetoscopy in a PFC environment, fetal endoscopy was performed before and after amniotic fluid exchange with perfluorodecalin in 2 additional rabbits ( $\mathrm{n}=10$ fetuses) on day 27 of gestation. A 3-mm diameter, $30^{\circ}$ optical angle endoscope was introduced into the amniotic cavity after securing the access site and incision with a 5-0 polypropylene pursestring suture to avoid any leakage of intraamniotic fluid. The goal of the intervention was to access the amniotic space, direct the endoscope towards the mouth of the fetus, and advance it into the oral cavity and posterior pharynx in order to visualize the larynx. The surgeon in control of the camera documented visibility and ease of handling during the procedure. Episodes of bleeding and resulting changes in visual acuity were documented on video recording.

\section{Data Acquisition and Analysis}

PFC distribution was first recorded radiographically in the intact uterus and then in the explanted fetuses. To calculate fetal lung/body weight (FLBW) ratios, the lungs were removed through a transsternal thoracotomy, the trachea having been ligated above the bifurcation. The fetal body and the lungs were weighed on an electronic scale with a balance accuracy of 1/100 g (LC4801P, Sartorius AG, Göttingen, Germany). Subsequently, the fetal brain was removed and a $1-\mathrm{ml}$ tissue sample of maternal subcutaneous fat excised. All tissues were placed in airtight containers and frozen at $-20^{\circ} \mathrm{C}$ for later analysis of PFC content by gas chromatography using a GC17A Analyzer with a QP5000 mass spectrometer (Shimadzu Corp., Duisburg, Germany) and a HS40 headspace sampler (PerkinElmer Instruments, Rodgau-Jügesheim, Germany). External standard reference samples were created with $1,5,10$, and $50 \mathrm{ppm}$ PFOB injected into $0.5 \mathrm{~g}$ of turkey meat for comparison. 

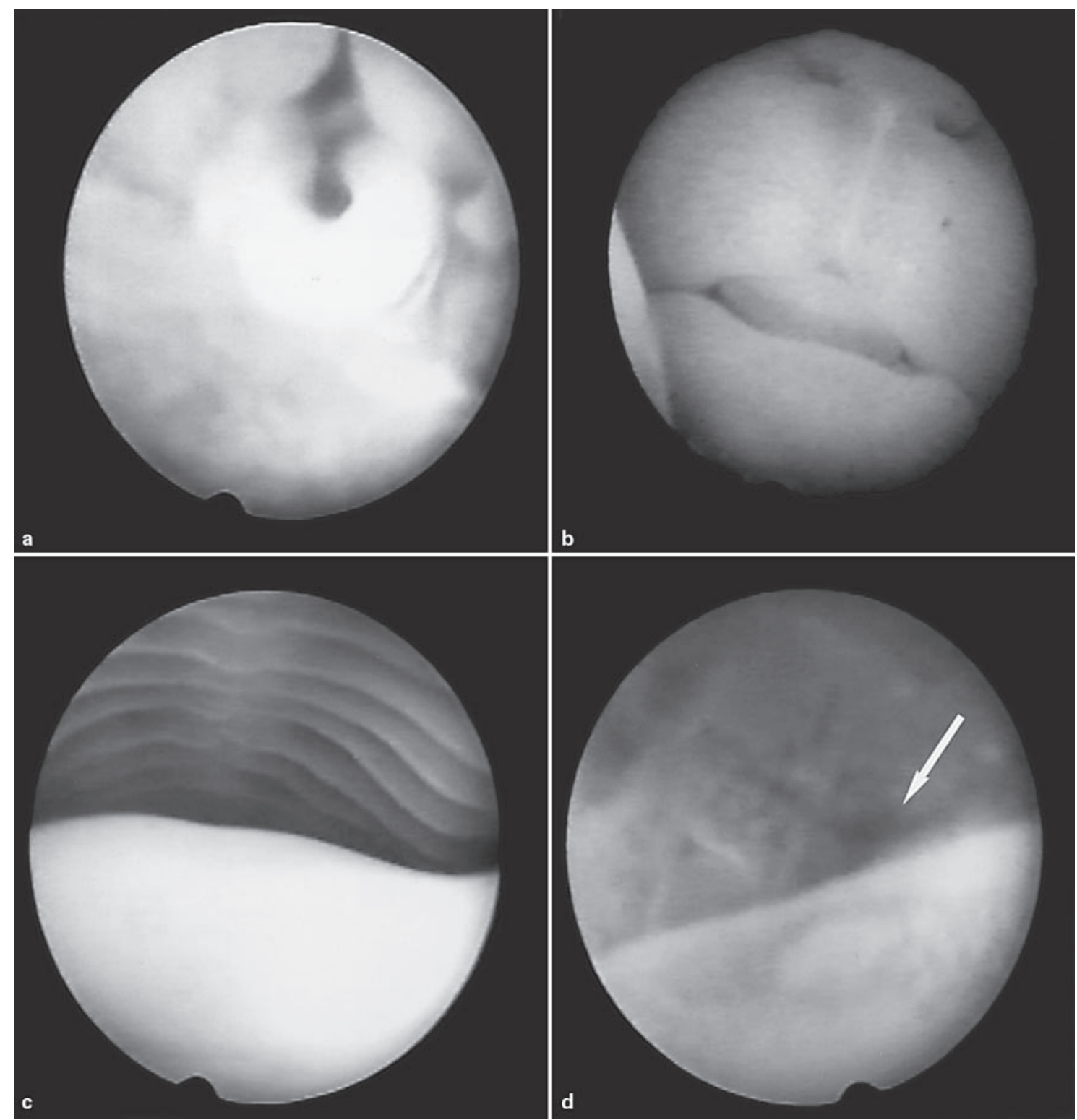

Fig. 1. Clear fetoscopic image of the glottis (a) using PFD as an endoscopic medium. This environment allows clear definition of the fetal rabbit's snout (b) and the oropharynx (c) with lighter tongue at the base of the image. In unreplaced amniotic fluid, bleeding from the tongue's base (arrow) blurs the optical definition in a corresponding view (d).

Sample temperature was $85^{\circ} \mathrm{C}$ heated for an interval of $45 \mathrm{~min}$. PFC content was determined by automated integration of the area under the PFC peak, with reference to the external samples.

\section{Statistics}

Survival rates between study groups were compared using the McNemar test. Mean heart rates were compared between the groups by t-test ( $\mathrm{p}<0.05$ was considered significant in all analyses). The FLBW ratio was compared between the groups using paired t-tests. All statistical analyses were performed with WinSTAT 2001.1 (R. Fitch Software, Staufen, Germany).

\section{Results}

\section{Fetoscopy}

Fetoscopy by the described method was feasible in amniotic fluid as well as in PFC. In both, the surgeon was able to advance the endoscope into the oral cavity and visualize the glottis (fig. 1a, image acquired in PFC medium) in a median number of only 1 attempt (range 1-3). While the naturally occurring debris from epithelial shed-

Fetal Diagn Ther 2005;20:359-365 
Fig. 2. Intraamniotic PFOB is documented on X-ray, filling the entire cavity without leakage into the surrounding structures.

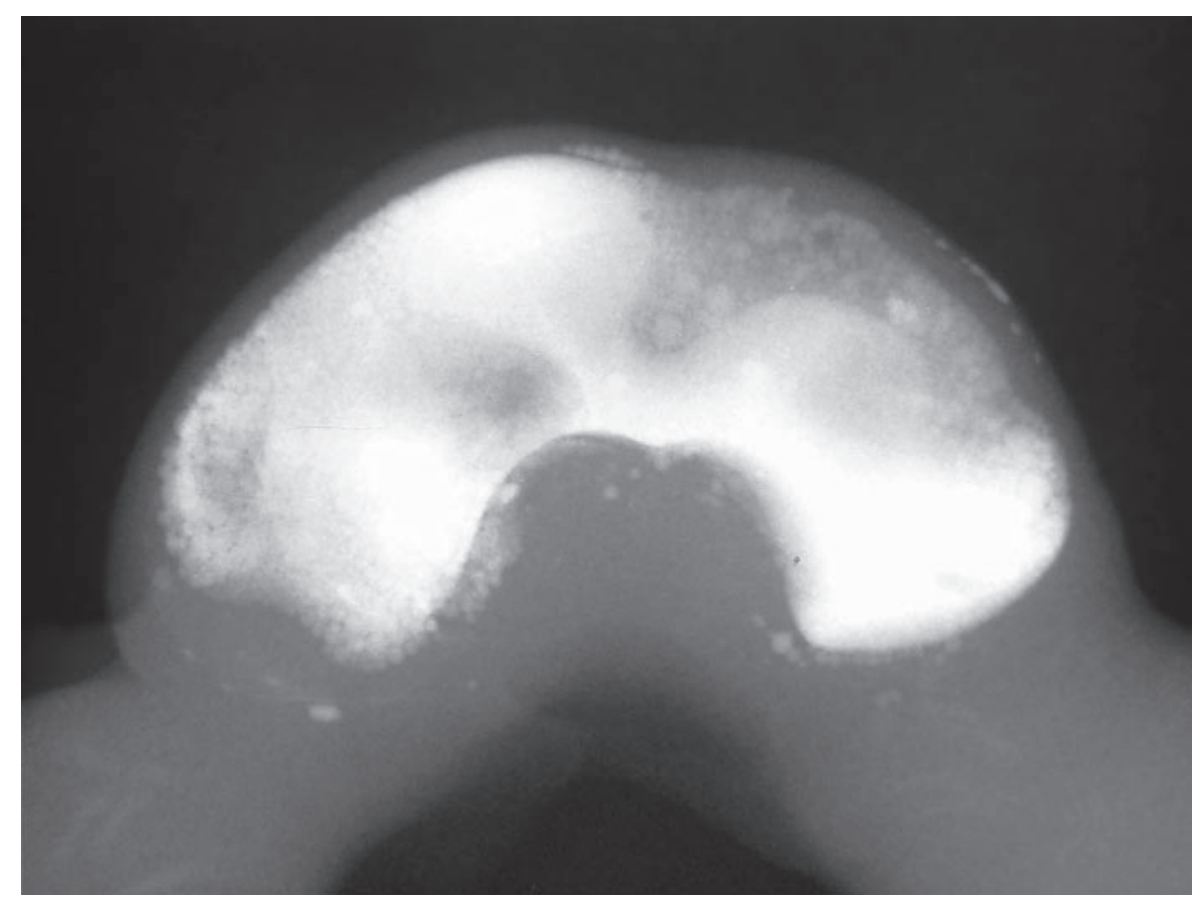

ding float around in the amniotic fluid and compromised clarity, using PFC as a medium provides a clear fetoscopic picture of the snout and the oropharynx (fig. $1 \mathrm{~b}$ and c, respectively). PFC penetrated and filled the pharynx before entering the mouth with the endoscope. In one occurrence before PFC exchange, bleeding into the amniotic fluid was encountered from the base of the tongue during endoscopic manipulation. Dispersion of blood into the aqueous amniotic fluid progressively blurred the endoscopic image and thereby hampered anatomic definition (fig. 1d) compared to the corresponding image after PFC exchange (fig. 1c). No bleeding was encountered during fetoscopy in the PFC environment. Mean fetoscopy times from endoscope placement to visualization of the glottis were similar in amniotic fluid (4 h $39 \mathrm{~min} \pm 81$ $\mathrm{min})$ and PFC ( $3 \mathrm{~h} 57 \mathrm{~min} \pm 44 \mathrm{~min})$.

\section{Viability of Fetuses during the Observation Period}

There was no statistically significant difference in immediate mortality between the study groups. After $6 \mathrm{~h}$, $12 / 17$ fetuses survived in the PFC group, compared to $12 / 17$ in the ES group $(p=0.56, \kappa=0.72)$, and $10 / 17$ in the control group $(\mathrm{p}=0.56, \mathrm{k}=0.75)$. In this context, a $\kappa$-index on the McNemar test $>0.7$ indicates a high degree of congruency between the cohorts, reflecting the fact that the entire litter of fetuses died in 3 does due to primary placental abruption. Clinically in these cases there was a sudden marked increase in uterine tone with peristaltic waves from the ovarian to the vaginal aspect in both uterine horns. Once manifest, increasing the continuous terbutaline infusion threefold did not stop the contractions. The other fetal deaths were sporadic in nature, without an obvious maternal or systematic cause.

The heart rates did not differ between the three groups $\left(\operatorname{PFC}\left(168 \pm 27 \mathrm{~min}^{-1}\right), \mathrm{ES}\left(174 \pm 23 \mathrm{~min}^{-1}\right)\right.$, and control group $\left(176 \pm 27 \mathrm{~min}^{-1}\right.$ (PFC vs. $\mathrm{ES} \mathrm{p}=0.136$; PFC vs. control $p=0.071$; ES vs. control $p=0.590)$. The first death was noted at $2.5 \mathrm{~h}$ in controls, at $3.5 \mathrm{~h}$ in the ES and at $4 \mathrm{~h}$ in the PFC group. During the ultrasound measurements, most viable fetuses with heart rates above 100/min displayed fetal movements.

\section{FLBW Ratios}

There was no significant difference in the mean FLBW ratio between the groups $[0.03161 \pm 0.00440$ in PFC, $0.03107 \pm 0.00406$ in EL $(p=0.74)$, and $0.03124 \pm$ 0.00365 in controls $(p=0.72)]$.

\section{Distribution of PFC in Does and Fetuses}

PFOB was used in the majority of experiments (11 out of 17) and had the advantage of being readily identified on X-ray because of its increased opacity. Although the physical and chemical properties vary slightly, no difference in handling was noted during the exchange 
Fig. 3 a-d. PFOB is found in the pharynx of 4 of 11 treated fetuses. In figure $3 d$, it is also seen in the esophagus, with a regularly interrupted column of PFOB as a possible sign of repeated swallowing of small boluses or esophageal motility.
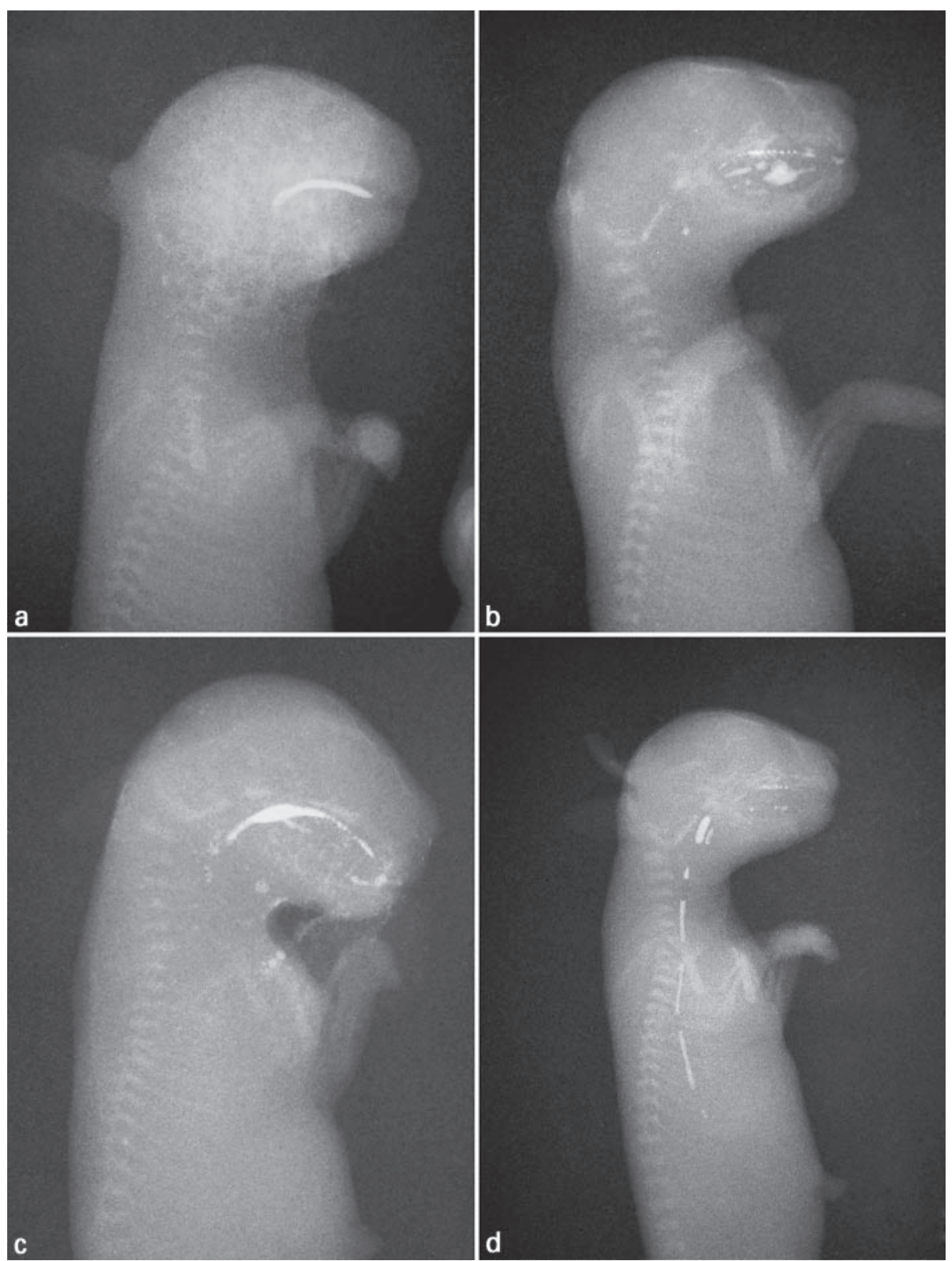

procedure. As documented on X-ray, the instilled intraamniotic PFC filled the entire cavity without leakage (fig. 2). PFOB was found on X-ray in the pharynx of 4/11 fetuses which had not undergone fetoscopy (fig. 3), but not in their lungs. One of these fetuses had PFOB documented both in the esophagus as well as the pharynx. Headspace gas chromatography did not detect any PFC of any kind in fetal lungs, brain, or maternal fatty tissues.

\section{Discussion}

Our investigation demonstrates that the exchange of amniotic fluid for PFC is feasible and does not affect the fetal heart rate or the survival of the fetuses. Fetoscopy seems to be of better quality when done in PFC as intrauterine medium, and visualization of the fetal rabbit larynx is easily performed. Our results further demonstrate that FLBW ratios do not differ between the treatment groups and that PFC is not detectable in any tissue after $6 \mathrm{~h}$ of instillation. The FLBW ratio was not expected to 
change because of lung growth in the short observation interval, but was evaluated as a sensitive means to detect any of the dense PFOB in the lower airways. Finally, the PFC did enter the pharynx of several fetuses, but did not reach the lower airways.

In this investigation, we also demonstrated that PFC might be a useful medium for fetal endoscopic surgery. Because PFC 'pushes' aqueous liquids and suspended debris to the top of the fluid level, a clear endoscopic image is obtained and conserved. In case of bleeding the lighter blood floats to the top, and image quality is not decreased to the same extent as in native amniotic fluid. Whether there was no bleeding observed in the PFC medium versus native amniotic fluid may be coincidental or perhaps due to a 'tamponade' effect of the dense PFC, which might lower the overall risk of bleeding. This should be the subject of future investigations, along with the systematic evaluation of different intraamniotic doses of PFC, gestational age at application, and delivery methods (bolus dosing, fractionated exchange as in this study, continuous perfusion). Another advantage of PFC is the lack of conductivity, allowing effective electrocauterization in the fluid-filled amniotic cavity. Other fetoscopic media have been tried, but have specific disadvantages: Insufflation of the amniotic cavity with $\mathrm{CO}_{2}$ causes respiratory acidosis [8]. Glycine is nonconductive [9], but is equally affected by suspended blood and debris. Because of its relative density, the fetus floats in the PFC environment and is therefore easily manipulated and accessible for endoscopic intervention. Due to the low surface tension of PFC, it penetrates small crevices and cavities easily, and thus facilitates the endoscopic access to the pharynx and other sites of difficult access.

The medical use of PFC has gained acceptance in a variety of experimental and clinical settings, including liquid ventilation, pulmonary drug delivery, vitreoretinal surgery, blood substitution, and contrast-enhanced imaging techniques [10-14]. However, experience so far with the prenatal and intraamniotic instillation of PFC is limited. In one investigation, the attempt to oxygenate rat fetuses transcutaneously by intraamniotic perfusion with PFC was unsuccessful [15]. In another study the perfusion of the amniotic cavity with oxygenated FC77 (a PFC liquid) resulted in no morphological alteration of the fetal lung, stomach or placenta [16]. In contrast, our investigation focused on the effect of PFC on functional aspects and the fetal viability as well as on the distribution of PFC within the maternofetal unit.

Increasing the amniotic pressure by infusing amniotic cavity with normal saline in goats has been shown to com- promise the fetal condition by lowering fetal $\mathrm{pH}$ and increasing fetal $\mathrm{pCO}_{2}$ [17]. Since filling the uterine cavity with PFC, a fluid of double the weight for volume compared to water, a higher static pressure and pressure gradient along the body axis could have adversely affected the circulation of the fetus. In a study in lambs [18], fetoplacental blood flow was preserved up to, and only significantly decreased beyond an intraamniotic pressure of $20 \mathrm{~mm} \mathrm{Hg}$. A pressure of $20 \mathrm{~mm} \mathrm{Hg}$ corresponds to water column of $26 \mathrm{~cm}$ or a PFC column of about $13 \mathrm{~cm}$. Due to the diameter of the amniotic cavity, the maximal pressure reached at the most dependent part of the uterus in rabbits should be far from that level, although this was not measured in this study. Furthermore, as the fetus and umbilical chord float to the top of the PFC-filled amniotic cavity, fetoplacental circulation should not be compromised by this mechanism. Since we found no difference in immediate mortality and heart rate of the fetuses between the three interventional groups, we believe that intrauterine PFC neither affects fetal circulation nor fetal survival.

After amnioinfusion with normal saline, increases in fetal plasma $\mathrm{Na}^{+}$and $\mathrm{Cl}^{-}$have been observed in sheep, indicating an uptake of these ions by the fetus [19]. In contrast, no significant shift of fluid or ions to the maternal compartment was observed after amnioinfusion with radiolabeled Hartmann's solution in sheep [20], even thought the ovine amnion is highly vascularized [21] compared to the monolayer epithelium found in humans and rabbits. While it is known that intrapulmonary PFC readily crosses the alveolar membrane and is found in fetal blood and tissues [6], no PFC was detected in the brains of fetuses exposed to intraamniotic PFC in our study. Also, PFC was not found in maternal fat and liver by headspace gas chromatography. Intraamniotic PFC therefore does not seem to pass into the maternal circulation in significant quantities within the study period.

Methylene blue was used to stain the amniotic fluid to facilitate amnio exchange with the nonstainable PFC. Although long-term effects of staining the amniotic fluid with methylene blue include small intestinal atresia and fetal death [22], no acute fetal toxicity is expected after the short exposure time in this study. Although somewhat more challenging due to the lack of clearly visual contrast, amnio exchange is feasible without prior staining.

Our initial hypothesis was that PFC in the amniotic space would be aspirated into the lungs by the fetus [23, 24]. Had this occurred, the denser PFC in the dependent pulmonary portions could hypothetically provide tissue stretch by gravitational forces as a potential noninvasive 
treatment for lung hypoplasia. Therefore, the experiment was carried out on day 27 of gestation, during early saccular stages of lung development, and not at day 22 as in previous models for fetoscopy in rabbits [25]. Although PFC was found in the pharynx of one third of the fetuses, it did not penetrate into the lungs over a period of $6 \mathrm{~h}$. Also, not all fetuses aspirated PFC into their upper airways despite the fact that no opiates were given to avoid compromising fetal respiratory movements [26]. Several reasons might account for the lack of PFC penetrating to lower airways. One is the predominant outflow of secreted fluid from the premature lungs, another the high resistance of the comparatively long and narrow trachea in fetal rabbits. A third and most likely cause was the short time the fetuses were exposed to the PFC environment. Provided there are longer periods of exposure, PFC might enter and remain within the lungs.

\section{Acknowledgments}

We would like to thank Till Olesch, $\mathrm{PhD}$, from Pharmpur $\mathrm{GmbH}$ in Augsburg for his scientific assistance in this project and the critical review of the manuscript. This research was supported by a grant from the University of Munich, Faculty of Medicine, Förderprogramm für Forschung und Lehre Nr. 156.

\section{References}

$\checkmark 1$ Harrison MR: Fetal surgery: Trials, tribulations, and turf. J Pediatr Surg 2003;38:275282.

-2 Harrison MR: Surgically correctable fetal disease. Am J Surg 2000;180:335-342.

3 Sydorak RM, Albanese CT: Minimal access techniques for fetal surgery. World J Surg 2003;27:95-102.

4 Flake AW: Surgery in the human fetus: The future. J Physiol 2003;547:45-51.

$\checkmark 5$ Lye SJ, Mitchell J, Nashman N, Oldenhof A, Ou R, Shynlova O, Langille L: Role of mechanical signals in the onset of term and preterm labor. Front Horm Res 2001;27:165-178.

-6 Shaffer TH, Wolfson MR, Greenspan JS, Hoffman RE, Davis SL, Clark LC Jr: Liquid ventilation in premature lambs: Uptake, biodistribution and elimination of perfluorodecalin liquid. Reprod Fertil Dev 1996;8:409-416.

7 Bennet L, Johnston BM, Gluckman PD: The central effects of morphine on fetal breathing movements in the fetal sheep. J Dev Physiol 1986;8:297-305.

-8 Luks FI, Deprest J, Marcus M, Vandenberghe $\mathrm{K}$, Vertommen JD, Lerut T, Brosens I: Carbon dioxide pneumoamnios causes acidosis in fetal lamb. Fetal Diagn Ther 1994;9:105-109.

-9 Ford WD, Cool JC, Byard R, Allanson M: Glycine as a potential window for minimal access fetal surgery. Fetal Diagn Ther 1997;12:145148.

10 Wolfson MR, Greenspan JS, Shaffer TH: Liquid-assisted ventilation: An alternative respiratory modality. Pediatr Pulmonol 1998;26: $42-63$.
11 Weiss DJ, Bonneau L, Liggitt D: Use of perfluorochemical liquid allows earlier detection of gene expression and use of less vector in normal lung and enhances gene expression in acutely injured lung. Mol Ther 2001;3:734745.

12 Arnold JH: Partial liquid breathing: Are we headed in the right direction? Crit Care Med 2000;28:1675-1676.

13 Crafoord S, Larsson J, Hansson LJ, Carlsson JO, Stenkula S: The use of perfluorocarbon liquids in vitreoretinal surgery. Acta Ophthalmol Scand 1995;73:442-445.

14 Huang MQ, Ye Q, Williams DS, Ho C: MRI of lungs using partial liquid ventilation with water-in-perfluorocarbon emulsions. Magn Reson Med 2002;48:487-492.

15 Czekelius P, Paschen W: Biochemical balance in the energy metabolism of fetal organs after perfusing the amniotic cavity with oxygenated fluorocarbon. Gynecol Obstet Invest 1987;24: 43-49.

16 Czekelius P, Kaduk B: Electron microscopy findings in fetal organs after perfusion of the amniotic cavity with oxygenated fluorocarbon. Arch Gynecol 1981;230:179-187.

- 17 Yoshihara H, Nemoto S, Suga H, Shimada N, Nishijima M, Shiotsu H, Satoh K: Effect of high amniotic fluid pressure on fetal circulation. J Perinatal Med 2000;28:221-227.

18 Skarsgard ED, Bealer JF, Meuli M, Adzick NS, Harrison MR: Fetal endoscopic ('Fetendo') surgery: The relationship between insufflating pressure and the fetoplacental circulation. $\mathbf{J}$ Pediatr Surg 1995;30:1165-1168.
19 Shields LE, Moore TR, Brace RA: Fetal electrolyte and acid-base responses to amnioinfusion: Lactated Ringer's versus normal saline in the ovine fetus. J Soc Gynecol Invest 1995;2: 602-608.

- 20 Evrard VA, Verbeke K, Peers KH, Luks FI, Lerut AE, Vandenberghe K, Deprest JA: Amnioinfusion with Hartmann's solution: A safe distention medium for endoscopic fetal surgery in the ovine model. Fetal Diagn Ther 1997; 12:188-192.

21 Albuquerque CA, Nijland MJ, Ross MG: Human and ovine amniotic fluid composition differences: Implications for fluid dynamics. J Matern Fetal Med 1999;8:123-129.

-22 Kidd SA, Lancaster PA, Anderson JC, Boogert A, Fisher CC, Robertson R, Wass DM: Fetal death after exposure to methylene blue dye during mid-trimester amniocentesis in twin pregnancy. Prenat Diagn 1996;16:39-47.

23 Rigatto H, Moore M, Cates D: Fetal breathing and behavior measured through a double-wall Plexiglas window in sheep. J Appl Physiol 1986;61:160-164.

24 Badalian SS, Fox HE, Chao CR, Timor-Tritsch IE, Stolar CJH: Fetal breathing characteristics and postnatal outcome in cases of congenital diaphragmatic hernia. Am J Obstet Gynecol

1994;171:970-976.
25 Papadopulos NA, Dumitrascu I, Ordonez JL, Decaluwe H, Lerut TE, Barki G, Deprest JA: Fetoscopy in the pregnant rabbit at midgesta-

tion. Fetal Diagn Ther 1999; 14:118-121.
26 Hasan SU, Lee DS, Gibson DA, Nowaczyk BJ, Cates DB, Sitar DS, Pinsky C, Rigatto H: Effect of morphine on breathing and behavior in fetal sheep. J Appl Physiol 1988;64:20582065. 